\title{
The structure of junctions between carbon nanotubes and graphene shells
}

Article

Accepted Version

Harris, P. J. F., Suarez-Martinez, I. and Marks, N. A. (2016)

The structure of junctions between carbon nanotubes and graphene shells. Nanoscale, 8. pp. 18849-18854. ISSN 20403364 doi: https://doi.org/10.1039/C6NR06461B Available at https://centaur.reading.ac.uk/67327/

It is advisable to refer to the publisher's version if you intend to cite from the work. See Guidance on citing.

Published version at: http://pubs.rsc.org/en/Content/ArticleLanding/2016/NR/c6nr06461b\#!divAbstract

To link to this article DOI: http://dx.doi.org/10.1039/C6NR06461B

Publisher: The Royal Society of Chemistry

All outputs in CentAUR are protected by Intellectual Property Rights law, including copyright law. Copyright and IPR is retained by the creators or other copyright holders. Terms and conditions for use of this material are defined in the End User Agreement.

\section{www.reading.ac.uk/centaur}

\section{CentAUR}

Central Archive at the University of Reading

Reading's research outputs online 


\section{Journal Name}

\section{COMMUNICATION}

\section{The structure of junctions between carbon nanotubes and graphene shells}

Received 00th January 20xx,

Peter J.F. Harris ${ }^{a}$, Irene Suarez-Martinez ${ }^{\mathrm{b}}$ and Nigel A. Marks ${ }^{\mathrm{b}}$

Accepted 00th January 20xx

DOI: $10.1039 / x 0 x \times 00000 x$

www.rsc.org/

Junctions between carbon nanotubes and flat or curved graphene structures are fascinating for a number of reasons. It has been suggested that such junctions could be used in nanoelectronic devices, or as the basis of three-dimensional carbon materials, with many potential applications. However, there have been few detailed experimental analyses of nanotube-graphene connections. Here we describe junctions between nanotubes and graphene shells in a material produced by passing a current through graphite. Transmission electron micrographs show that the junction angles are not random but fall close to multiples of $30^{\circ}$. We show that connections with these angles are the only ones which are consistent with the symmetry of the hexagonal lattice, and molecular models show that a continuous lattice requires the presence of large carbon rings at the junction. Some of the configurations we propose have not been previously considered, and could be used to construct new kinds of three-dimensional carbon architecture. We also discuss the possible formation mechanism of the junctions.

Carbon nanotubes ${ }^{1}$ and graphene ${ }^{2}$ are two of the most exciting nanomaterials to have been discovered in the past 25 years. The idea of joining nanotubes to flat graphene or to curved graphene structures has attracted much interest. For example, it has been suggested that such junctions could be used as the basis of three-dimensional carbon architectures such as "pillared" graphene, in which parallel graphene sheets are separated by short nanotubes ${ }^{3}$. Such materials might have interesting mechanical 4 or thermal 5 properties or have applications in hydrogen storage ${ }^{3}$ or gas separation ${ }^{6}$. However,

\footnotetext{
a. Electron Microscopy Laboratory, Department of Chemistry, J.J. Thomson Building, University of Reading, Whiteknights, Reading RG6 6AF, UK.

E-mail: p.j.f.harris@reading.ac.uk

b. Nanochemistry Research Institute, Department of Physics \& Astronomy, Curtin

University, Perth, WA 6102, Australia.

E-mail:1.Suarez-Martinez@curtin.edu.au; N.Marks@curtin.edu.au
}

Electronic Supplementary Information (ESI) available: Details of the method used to prepare the carbon and models of $30^{\circ}$ and $60^{\circ}$ junctions. See DOI: $10.1039 / x 0 x x 00000 x$ synthesising covalently-bonded nanotube-graphene junctions has proved extremely challenging. While it is possible to grow nanotubes vertically on graphene using chemical vapor deposition (CVD) ${ }^{7-9}$, in most cases the nanotubes in these materials are not covalently bonded to the graphene. The only evidence for seamless, covalent bonding between nanotubes and graphene in such materials is work by Tour et al. ${ }^{10}$, where atomic resolution transmission electron microscopy appeared to show the presence of heptagonal rings at the junctions. An alternative, albeit less controlled, way of creating carbon materials containing nanotubes joined to graphene structures is to pass an electric current through graphite or through fewlayer graphene ribbons. The aim of the present paper is to analyse nanotube-graphene junctions formed in this way.

Evidence that the structure of graphite or graphene nanoribbons could be transformed by the passage of an electric current was first presented in three papers published in 2009 1113. In two of these 11,12, the specimens used were graphite "nanoribbons" and the transformations were observed directly using in situ Joule heating inside a TEM. In the third, by one of the present authors ${ }^{13}$, the transformed structures were observed in bulk samples of graphite through which a current had been passed. The structural transformation involves the formation of a carbon material with a highly irregular edge morphology, displaying many unusual features, including the nanotube-graphene junctions which are the subject of this paper. Several subsequent studies have been carried out into the phenomenon 14-21. Interestingly, when the starting materials are graphite nanoribbons, or few-layer graphene, the transformed material is usually made up of single-layer graphene, while using bulk graphite as the starting material generally results in bilayer structures. The reasons for this are not fully understood. There is some disagreement about the mechanism of the transformation. Some authors have discussed the process in terms of sublimation and edge reconstruction of flat graphene 11,12,18-21. An alternative 

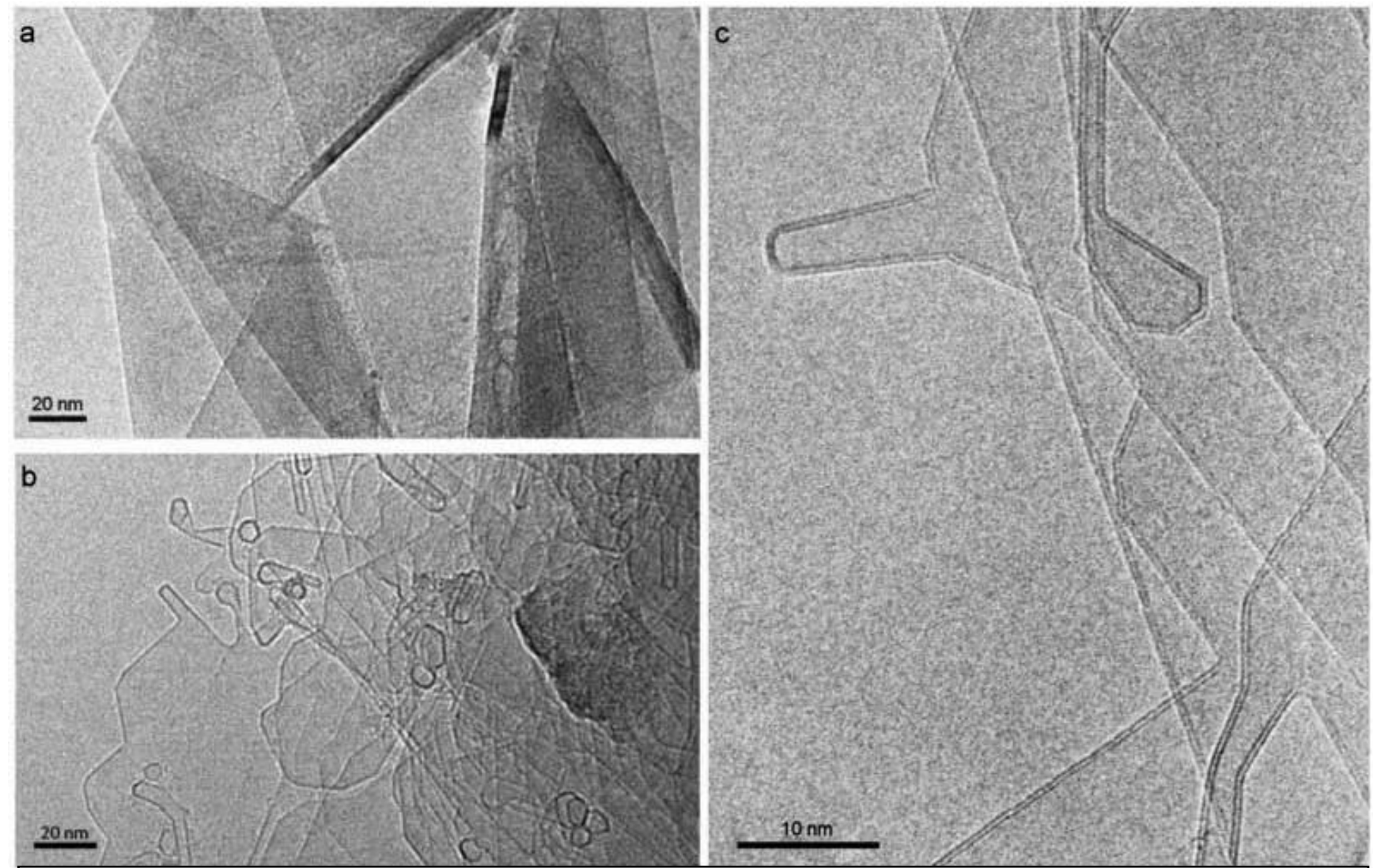

Figure 1 (a) Transmission electron micrograph of carbon from fresh graphite rod. (b) Region with disordered structure following passage of current. (c) Higher magnification image of transformed region showing bilayer structure.

explanation is that the transformation actually involves a change from a flat to a three-dimensional structure 14-17. Supporting evidence for the latter hypothesis has been obtained by using a combination of high-angle annular darkfield imaging and electron energy loss spectroscopy in the scanning transmission electron microscope ${ }^{16}$.

In order to pass a current through small samples of graphite, we used a commercial arc-evaporator, which is normally used for carbon-coating specimens for scanning electron microscopy. In this unit the electrodes are graphite rods, one of which is thinned to a diameter of approximately $1.4 \mathrm{~mm}$ at the point of contact. Following evaporation, the thinned carbon rod was found to have slightly shortened, and a small deposit was formed in the area where the two rods made contact (see Supporting Information for more details). This was collected and ground under isopropyl alcohol, and droplets of the suspension were pipetted onto lacey carbon TEM grids. Material collected from the fresh graphite rods was also imaged by TEM for comparison. Conventional TEM imaging was carried out using a JEOL 2010 microscope, with a point resolution of $0.19 \mathrm{~nm}$, operated at an accelerating voltage of $200 \mathrm{kV}$. Experiments carried out with the samples studied here showed that visible damage occurred only after about 2 mins exposure to a beam with a current density of $15 \mathrm{pA} \mathrm{cm}^{-2}$. Care was taken not to expose the carbon to an electron beam for longer than this time.
Figure 1a shows material from the fresh graphite rod. As expected, this consists mainly of flat crystallites, ranging from a few $100 \mathrm{~nm}$ to about $5 \mu \mathrm{m}$ in size. Lattice imaging showed that these crystallites contained up to 100 layers. The carbon collected from the graphite rods following passage of a current contained some "normal" graphite, but this was accompanied by many regions which had a very different morphological appearance. A typical transformed area is shown in Figure $1 \mathrm{~b}$. As can be seen, the outline of the structure in this area of the material is more irregular than in the fresh graphite, with many curved and unusually-shaped features. Higher resolution images of the transformed carbon show that it largely consists of bilayer graphene, as can be seen in Figure 1c. The interplanar spacing in the bilayer structures was generally in the range 0.38 $-0.40 \mathrm{~nm}$, somewhat larger than the spacing for graphite $(0.335$ $\mathrm{nm})$.

Examples of nanotube-graphene junctions can be seen in Figure $1 \mathrm{~b}$ and $1 \mathrm{c}$, and further examples are shown in Figure 2. A large number of images of the junctions were recorded, and the angles of the junctions measured. When making these measurements, it must be recognised that the correct angle will only be observed if the junction is precisely perpendicular to the electron beam, and this will not always be the case. Nevertheless, it was clear that the measured angles were not randomly distributed. This can be seen in Figure $2 a$, which shows the distribution of angles from 100 such junctions. There are peaks at angles of $30^{\circ}, 60^{\circ}, 90^{\circ}, 120^{\circ}, 150^{\circ}$, with the larger 
a
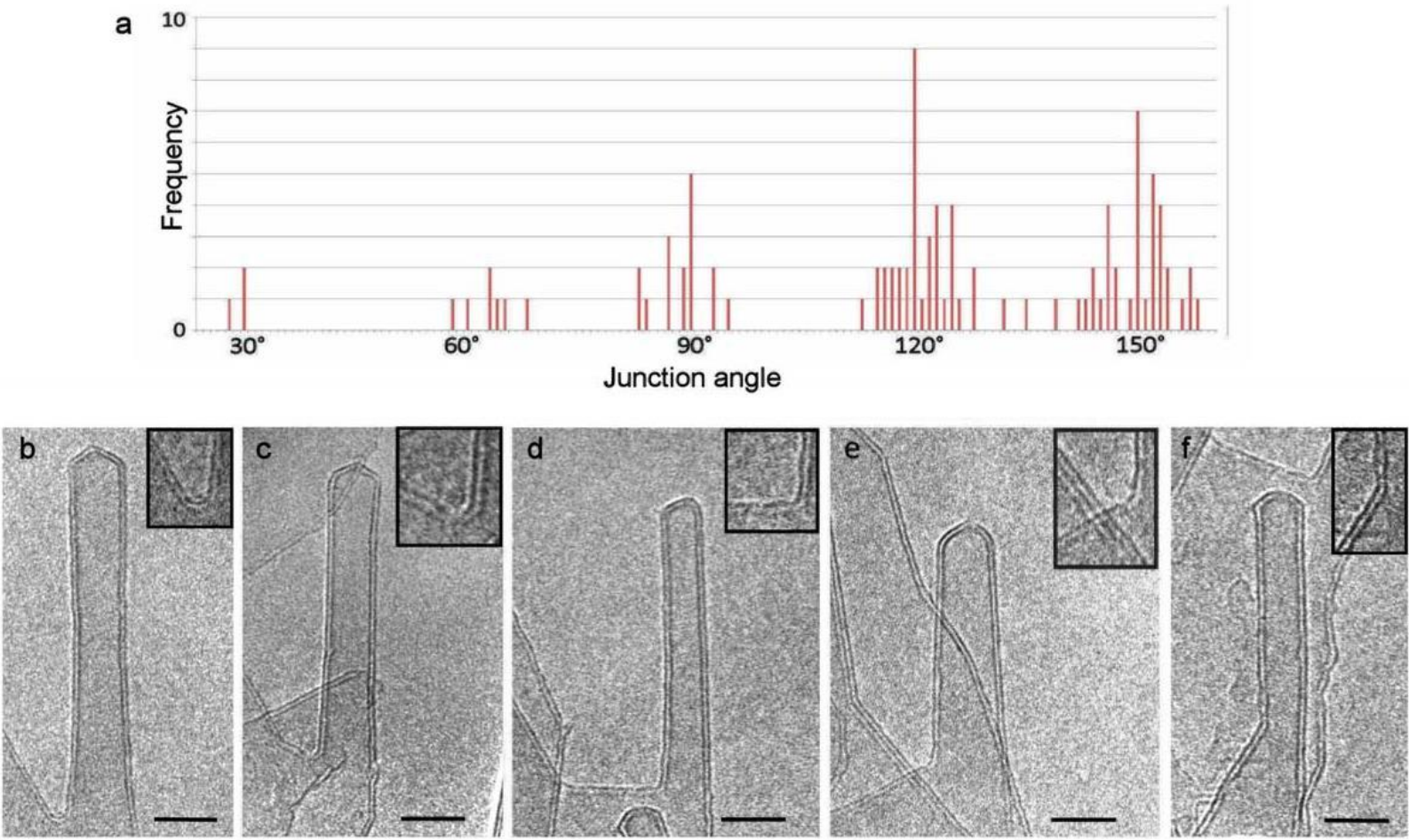

Figure 2 (a) Chart showing distribution of angles in 100 nanotube-graphene junctions. (b) - (f) Micrographs showing junctions with angles of approximately $30^{\circ}, 60^{\circ}, 90^{\circ}, 120^{\circ}$ and $150^{\circ}$. Scale bar $5 \mathrm{~nm}$. Insets show enlarged images of junctions.

angles being rather more common than the smaller ones. In the case of the junction angles clustered around $60^{\circ}$, the highest frequency is closer to $65^{\circ}$ degrees than $60^{\circ}$. This is simply a consequence of the small number of $60^{\circ}$ junctions we observed (7 in total). For the larger junction angles the frequency distributions tend more closely towards Gaussian. Micrographs showing junctions with angles close to $30^{\circ}, 60^{\circ}, 90^{\circ}, 120^{\circ}$ and $150^{\circ}$ are shown in Figure $2 \mathrm{~b}-\mathrm{f}$.

We now discuss the possible structures of the junctions, and the reasons why certain angles are apparently favoured. For simplicity we consider single-layer graphene in our simulations, although the experimental structures were bilayer. This does not affect the arguments. A generic nanotube-graphene junction is depicted schematically in Figure 3a. The angle between the tube and the folded layer, $\alpha$, may take any value in a continuous model. However, when the atomistic structure of graphene is considered, the chirality of the tube dictates the topology of folded graphene and vice-versa. Consideration of the hexagonal network shows that the only possible junction angles are multiples of $30^{\circ}$, as seen in our experimental images. The argument holds to any chirality as we will demonstrate but we will describe it in detail for the example of the possible folded graphenes with a $(2,3)$ nanotube. The unrolled $(2,3)$ nanotube is depicted in dark grey on the graphene lattice in Figure $3 \mathrm{~b}$. The chiral vector $(2,3)$ is depicted in red and is perpendicular to the translational vector $(8,-7)$ depicted in blue. The chiral vector and its rotated equivalents are depicted in green for each possible folded graphene. When folded through the dashed black line, two of the green vectors will overlap, and the folded graphene will present an edge (marked by a black dot and thick black line) which will atomistically match with the nanotube.

This geometrical reasoning can be generalized as for the front and the back of the folded graphene to atomistically match with the nanotube, the in-plane vectors of the graphene sheet (marked in green in Figure 3a) and its perpendicular vector must be in the same lattice direction as the chiral vector and translational vector of the tube respectively (marked in red and blue in Figure 3a). A perfect graphene layer has a $\mathrm{C} 6$ symmetry axis, meaning it repeats upon rotation every $60^{\circ}$ degrees rotation. Therefore, the angle in which a nanotube connects with folded graphene can only be $\mathrm{n} \times 30^{\circ}, \mathrm{n}$ being an integer from 1 to 5 .

While the hexagonal network of graphene explains the observed angles, details of the topology of the junction (depicted by a black dot in Figure 3) can only be hypothesised. Molecular models for the different junctions are constructed by bonding a capped nanotube to a folded sheet of graphene. For simplicity in the atomistic construction an armchair tube is chosen and therefore the graphene sheet is folded along the zigzag directions. We chose a $(5,5)$ nanotube as it is easily capped by half fullerene. All models are optimised using the Universal Force Field ${ }^{22}$. Models for junctions of $150^{\circ}, 120^{\circ}$ and $90^{\circ}$ are shown in Figure 4 . All junctions can be modelled by a single non-hexagonal ring: $150^{\circ}$ by a heptagon, $120^{\circ}$ by an octagon, $90^{\circ}$ by a nonagon, $60^{\circ}$ by a decagon and $30^{\circ}$ by a hendecagon. The junction of $150^{\circ}$ can only be modelled by a 
a)

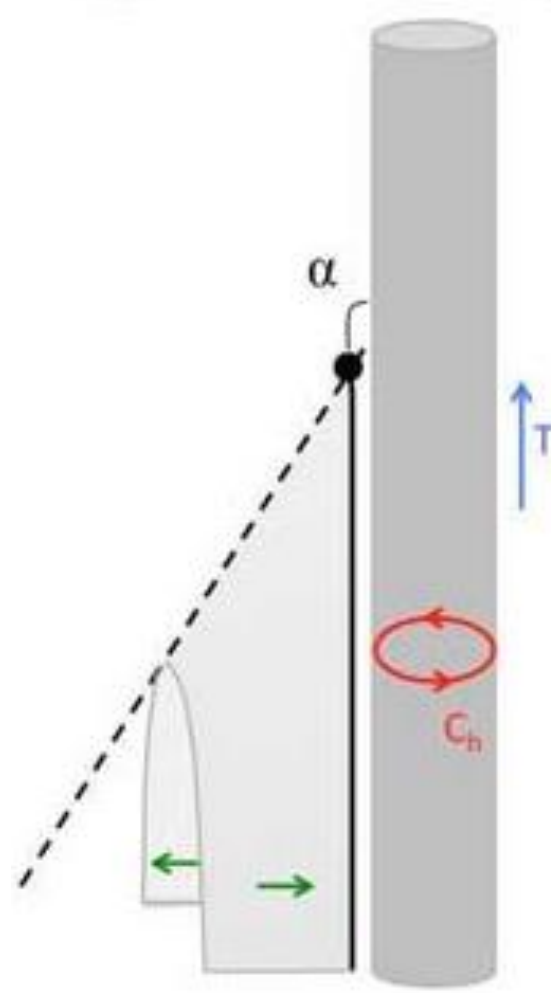

b)

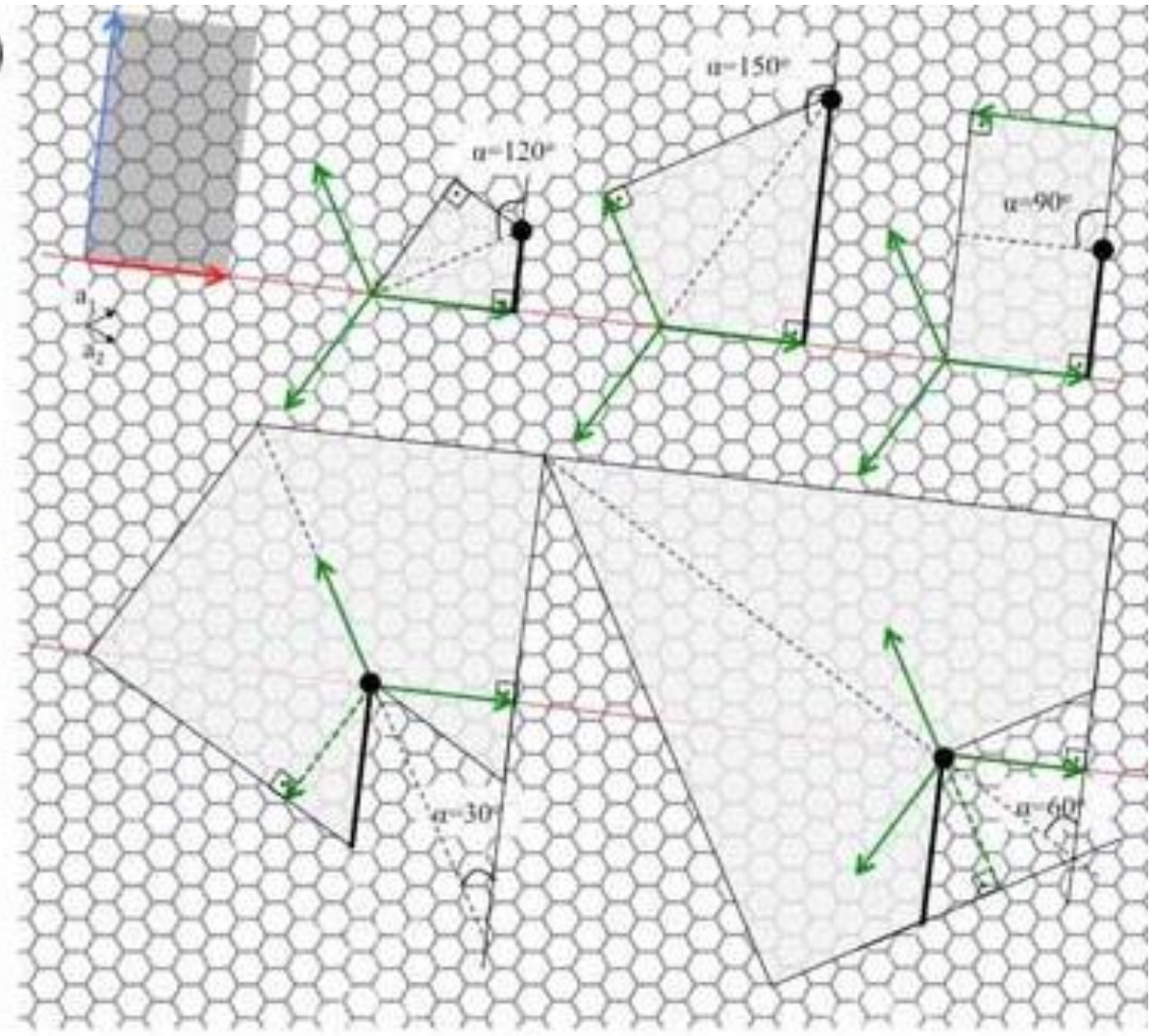

Figure 3 (a) Continuous model of a nanotube-graphene junction. The nanotube is depicted in dark grey and its chiral vector, $\mathrm{Ch}$, which is depicted in red, defines it. The tube axis is parallel to the transitional vector, $\mathrm{T}$, depicted in blue, and it is perpendicular to the chiral vector. A folded graphene is depicted in light grey where a dashed line indicates the fold. (b) Example of the $(2,3)$ nanotube and its possible folded graphene junctions.

single heptagon. This junction appears to be very similar to certain kinds of nanotube cap, first identified by lijima et al. ${ }^{23}$, in which a flattened cone is joined to a short, closed, tube. lijima and colleagues proposed that a single heptagonal ring was present at the junction, and we believe this is also the case in the $150^{\circ}$ junctions seen here. All other junctions can be modelled by combinations of heptagons or by combinations of pentagons and octagons. For example, a $90^{\circ}$ junction can be produced by a single nonagon, 3 adjacent heptagons or 2 octagons and a pentagon. The schematics in Figure 4c show how transformation between these combinations may occur. Similar transformations occur for all other junctions. Models of $30^{\circ}$ and $60^{\circ}$ junctions are given in Supplementary Information.

The $120^{\circ}$ and $90^{\circ}$ junctions resemble $\mathrm{Y}$ - and T- nanotubenanotube junctions, respectively. Combinations of double heptagons have been proposed previously by Chernozatonskii in models of $120^{\circ}$ nanotube $\mathrm{Y}$-junctions ${ }^{24}$. Li and colleagues have described models in which nonagons occur at $90^{\circ}$ junctions between very narrow nanotubes and flat bilayer graphene (separated by the normal interlayer distance of approximately $0.34 \mathrm{~nm}$ ) ${ }^{21}$, while Menon and Srivastava employed 3 adjacent heptagons in models of nanotube Tjunctions ${ }^{25}$. All those combinations are in agreement with our model of nanotube-graphene junctions.
While there are similarities between the junctions in the present work and nanotube-nanotube connections, they are different to nanotube-graphene junctions which have previously been considered ${ }^{3-6,26,27}$. The structures presented here can be thought of as tubes joined to folded graphene structures whose edges constitute "semi-tubes". However previous nanotube-graphene junctions models have envisaged tubes joined perpendicularly to flat graphene. The junctions have generally contained 6 heptagons distributed around the base of the tube. It has proved very difficult to synthesise threedimensional architectures containing such junctions. With the exception of the work by Tour et al. ${ }^{10}$, mentioned above, there is little clear experimental evidence for such junctions. A threedimensional carbon prepared by Dai and colleagues ${ }^{28}$ did seem to have seamless junctions, but these were found to have angles of $135^{\circ}$, apparently due to funnel-like structures at the connecting points. The present work shows that there are many types of nanotube-graphene connection, in addition to the usually considered $90^{\circ}$ one. It would be an interesting challenge for theoreticians to come up with new three-dimensional architectures containing some of the new junctions described here. 


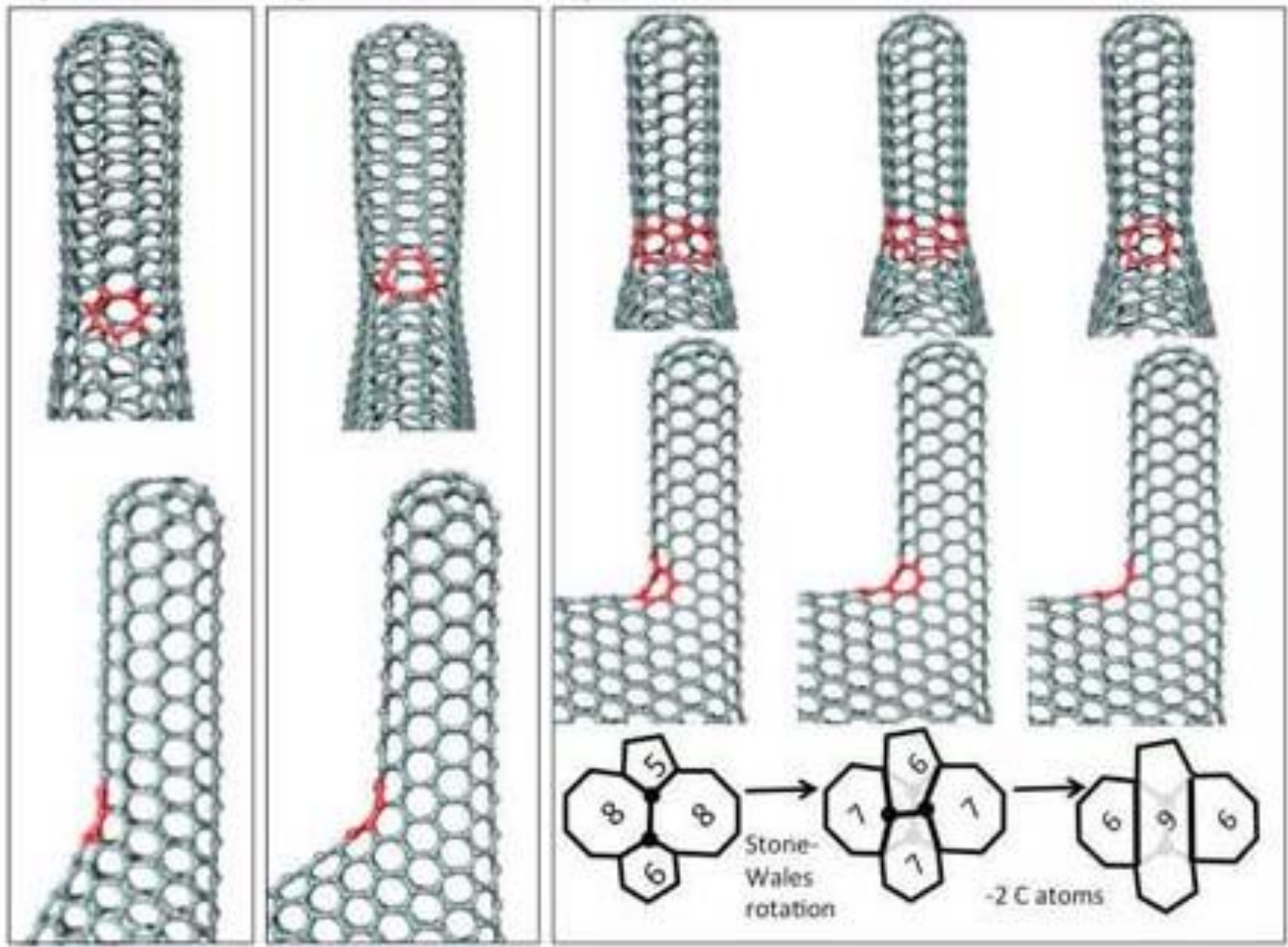

Figure 4 Atomistic models for junction of a $(5,5)$ carbon nanotube with folded graphene. (a) Single heptagon in a $150^{\circ}$ junction. (b) Single octagon in a $120^{\circ}$ junction. (c) Three possible atomistic descriptions of a $90^{\circ}$ junction.

We now consider the possible formation mechanism of the nanotube-graphene junctions. As discussed in previous papers, we believe that the restructuring of graphite which occurs on passage of a current involves an opening or separation of the layers, apparently beginning at folded edges, as illustrated schematically in Figure 5a. As supporting evidence for this mechanism, we have published images of graphite fragments which display the early stages of the process (see for example Figure $8 \mathrm{c}$ in ref. 16). However, the reasons why the passage of a current should produce such a transformation remain unclear. Concerning the formation of nanotubes attached to larger graphene structures, it is possible that these nucleate at the pentagonal rings which are believed to occur where zigzag and armchair edges meet, as shown in Figure $5 b^{29}$. A possible mechanism is illustrated schematically in Figure $5 \mathrm{c}$. This shows three stages in the transformation of an initially flat, 4-layer graphene structure, with closed edges, into a three-dimensional structure in which a bilayer nanotube is joined to a graphene shell with bilayer walls. As the opening process begins, the presence of the pentagon impedes the separation of the layers, forcing the hexagons around it to adopt a hemispherical morphology which evolves into a
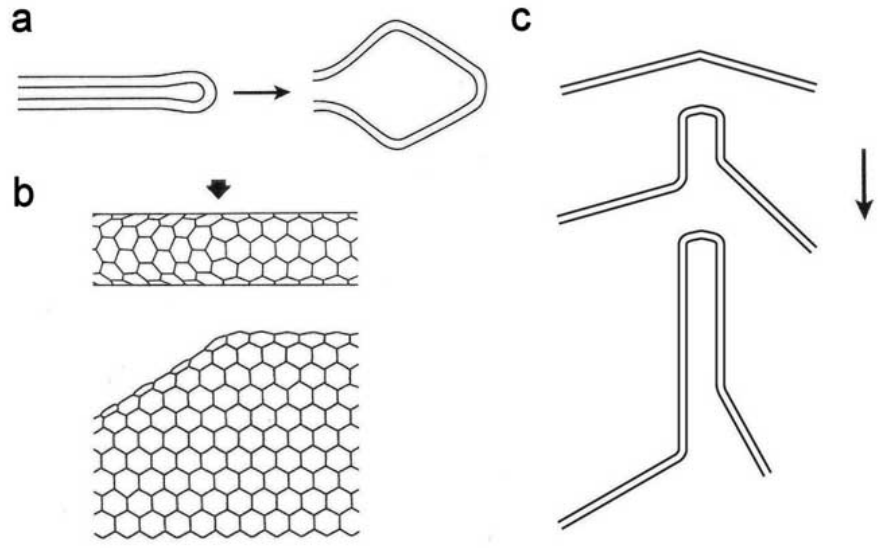

Figure 5 Diagrams illustrating possible formation mechanism of nanotube-graphene junctions. (a) Sketch showing suggested mechanism for transformation of folded graphene sheets into hollow structure, (b) drawings of folded monolayer graphene, showing the region where zig-zag edge meets armchair edge. For simplicity, only the upper layer is shown in the plan-view drawing. Arrow indicates position of pentagonal ring, (c) sketches showing how a nanotube-graphene junction might evolve from the point where zig-zag and armchair edges meets. Arrow shows direction of "retreating front". 
nanotube cap. As the folded graphene layers either side of the incipient cap become separated and expand perpendicular to the plane of the paper, a short nanotube is left behind. Processes rather similar to this can be seen in the videos which accompanied reference 12 , although in this case the final structures are monolayer rather than bilayer. Further in situ studies of this kind would be very valuable in understanding the mechanism in greater detail.

The method we have used in this study to transform the structure of graphite is very uncontrolled. It is possible that a more controlled application of a current could be used to build structures in which nanotubes are joined to graphene in a more organised way. One approach would be to create a patterned graphene using techniques such as those discussed by Li et al. ${ }^{30}$ and then apply an electric current to specific points in the pattern to effect a localised transformation. In this context it is interesting to recall work by Suenaga and colleagues ${ }^{31}$. These workers used in situ techniques inside a TEM to show that nanotubes could be "plumbed" together by the passage of a current. Although this process is not the same as that which produced the junctions described here, it does show that the application of an electric current can be used at the nanoscale to create new carbon architectures in a controlled way.

\section{Conclusions}

In summary, we have presented an analysis of junctions between nanotubes and graphene shells in a carbon material produced by passing a current through graphite. Transmission electron microscopy of the junctions has shown that the junction angles generally fall close to multiples of $30^{\circ}$. A consideration of the hexagonal graphene network has shown that such angles are the only ones which are consistent with a continuous lattice. It has also been demonstrated that all junctions can be modelled by a single non-hexagonal ring at the point of contact between the nanotube and the graphene shell, although configurations involving multiple non-hexagonal rings are also possible. The junctions described here could form the basis for new three-dimensional carbon architectures with many potential applications.

\section{References}

1 P. J. F. Harris, Carbon Nanotube Science, Cambridge University Press, 2009.

2 J. H. Warner, F. Schaffel, M. H. Rummeli and A. Bachmatiuk, Graphene: Fundamentals and emergent applications, Elsevier, 2013.

3 G. K. Dimitrakakis, E. Tylianakis and G. E. Froudakis, Nano Letters, 2008, 8, 3166-3170.

4 R. Shahsavari and N. Sakhavand, Carbon, 2015, 95, 699-709.

5 J. Shi, Y. Dong, T. Fisher and X. Ruan, J. Appl. Phys., 2015, 118, 044302.

6 R. P. Wesołowski and A. P. Terzyk, Physical Chemistry Chemical Physics, 2011, 13, 17027-17029.
7 F. Du, D. S. Yu, L. M. Dai, S. Ganguli, V. Varshney and A. K. Roy, Chemistry of Materials, 2011, 23, 4810-4816.

8 J. Lin, C. Zhang, Z. Yan, Y. Zhu, Z. Peng, R. H. Hauge, D. Natelson and J. M. Tour, Nano Letters, 2013, 13, 72-78.

9 J. G. Zhao, B. Y. Xin, H. Yang, Q. L. Pan, Z. P. Li and Z. J. Liu, New Carbon Materials, 2016, 31, 31-36.

10 Y. Zhu, L. Li, C. G. Zhang, G. Casillas, Z. Z. Sun, Z. Yan, G. D. Ruan, Z. W. Peng, A. R. O. Raji, C. Kittrell, R. H. Hauge and J. M. Tour, Nature Communications, 2012, 3, 1225.

11 X. T. Jia, M. Hofmann, V. Meunier, B. G. Sumpter, J. CamposDelgado, J. M. Romo-Herrera, H. Son, Y. P. Hsieh, A. Reina, J. Kong, M. Terrones and M. S. Dresselhaus, Science, 2009, 323 , 1701-1705.

12 J. Y. Huang, F. Ding, B. I. Yakobson, P. Lu, L. Qi, and J. Li, Proceedings of the National Academy of Sciences of the United States of America, 2009, 106, 10103-10108.

13 P. J. F. Harris, Journal of Physics: Condensed Matter, 2009, 21, 355009.

14 P. J. F. Harris, Philosophical Magazine, 2011, 91, 2355-2363.

15 P. J. F. Harris, Carbon, 2012, 50, 3195-3199.

16 P. J. F. Harris, T. J. A. Slater, S. J. Haigh, F. S. Hage, D. M. Kepaptsoglou, Q. M. Ramasse and R. Brydson, Nanotechnology, 2014, 25, 465601.

17 P. J. F. Harris, Carbon, 2016, 107, 132-137.

18 L. Qi, J. Y. Huang, J. Feng and J. Li, Carbon, 2010, 48, 23542360.

19 A. Barreiro, F. Börrnert, M. H. Rümmeli, B. Büchner and L. M. K. Vandersypen, Nano Letters, 2012, 12, 1873-1878.

20 E. Cruz-Silva, X. T. Jia, H. Terrones, B. G. Sumpter, M. Terrones, M. S. Dresselhaus and V. Meunier, ACS Nano, 2013, 7, 2834-2841

21 J. S. Qi, J. Y. Huang, J. Feng, D. N. Shi and J. Li, ACS Nano, 2011, 5, 3475-3482.

22 A.K. Rappé, C. J. Casewit, K. S. Colwell, W. A. Goddard III, W. M. Skiff, J. Amer. Chem. Soc., 1992, 114, 10024-10035.

23 S. lijima, T. Ichihashi and Y. Ando, Nature, 1992, 356, 776778.

24 L.A. Chernozatonskii, Physics Letters A, 1992, 172, 173-176.

25 M. Menon and D. Srivastava, Physical Review Letters, 1997, 79, 4453-4456.

26 T. Matsumoto and S. Saito, Journal of the Physical Society of Japan, 2002, 71, 2765-2770.

27 D. Baowan, B. J. Cox and J. M. Hill, Carbon, 2007, 45, $2972-$ 2980.

28 Y. Xue, Y. Ding, J. B. Niu, Z. H. Xia, A. Roy, H. Chen, J. Qu, Z. L. Wang and L. M. Dai, Sci. Adv., 2015, 1, 1400198.

29 Z. Liu, K. Suenaga, P. J. F. Harris and S. lijima, Physical Review Letters, 2009, 102, 015501

30 J. Feng, W. B. Li, X. F. Qian, J. S. Qi, L. Qi and J. Li, Nanoscale, 2012, 4, 4883-4899.

31 C. Jin, K. Suenaga and S. lijima, Nature Nanotechnology, 2008, 3, 17-21. 